EXTENDED REPORT

\title{
Estimation of inflammation by Doppler ultrasound: quantitative changes after intra-articular treatment in rheumatoid arthritis
}

\author{
L Terslev, S Torp-Pedersen, E Qvistgaard, B Danneskiold-Samsoe, H Bliddal
}

Ann Rheum Dis 2003;62:1049-1053

See end of article for authors' affiliations

\section{Correspondence to:} Dr L Terslev, The Parker Institute, Department of Rheumatology,

Frederiksberg Hospital, DK 2000 Frederiksberg,

Denmark: lene.terslev@

fh.hosp.dk

Accepted 15 March 2003

\begin{abstract}
Objective: To evaluate the use of ultrasound, including quantitative Doppler analysis of synovial vascularisation, before and after intra-articular treatment with glucocorticosteroids in patients with rheumatoid arthritis (RA).

Methods: 51 patients with RA were followed prospectively after an intra-articular glucocorticosteroid injection. Disease modifying antirheumatic drug treatment was kept unchanged and no further injections given in this observation period. At baseline, disease activity was estimated clinically by target join pain on a $100 \mathrm{~mm}$ visual analogue scale, on which the target joint was scored 0-3 for swelling and tenderness, and by ultrasound measurements of grey scale pixels, colour Doppler pixels, and the spectral Doppler resistive index (RI) as indicators of synovial swelling and inflammation. Affer four weeks, the measurements were repeated on the same joint. An observer unaware of the sequence and patient number evaluated the ultrasound images.

Results: At one month follow up after the glucocorticosteroid injection, a marked decrease in the fraction of colour pixels was seen in $41 / 51$ patients (Student's $t$ test $p<0.001$ ). Correspondingly, the RI increased indicating a diminished flow to the synovium (Student's $t$ test $p<0.01$ ). Both the fraction of colour pixels and the RI values corresponded with the clinical evaluation and with the subjective effect of the treatment. The synovial membrane volume estimated by total amount of pixels showed a significant decrease by $31 \%$ after treatment.

Conclusion: Ultrasound-Doppler seems to be a promising tool for the estimation of synovial activity in arthritis. After intra-articular glucocorticosteroid, changes in RI and fraction of colour pixels may both be used as quantitative measurements of the blood flow.
\end{abstract}

n rheumatological research non-invasive imaging methods such as magnetic resonance imaging (MRI) and ultrasound (US) have been proposed for the detection and grading of rheumatic inflammatory lesions as they have the potential to provide objective analysis of the disease without ionising radiation. ${ }^{1}$ Among the two imaging modalities, US has the potential of becoming a tool used in daily rheumatology practice as it is already accessible in many outpatient clinics, has no contraindications, and poses no problems of patient compliance. Studies have shown that US is comparable with MRI in detecting synovitis in rheumatoid arthritis (RA). ${ }^{12}$ Macroscopically the synovial membrane in RA joints is greatly increased and reflects vascular congestion, oedema and cellular infiltration as well as synovial lining hyperplasia and pannus tissue formation. ${ }^{3}$ US may visualise the vascularity in the pannus and the hyperaemic synovium by the use of Doppler, and several studies have indicated that power and colour Doppler ultrasonography can detect treatment response. ${ }^{4-7}$ The evaluation of the degree of inflammation and the treatment response has in general been based on a semiobjective scoring system grading the disease activityfor example, from 0 to $3,{ }^{45}$ while a more quantitative analysis of the amount of pixels with signs of vascular activity has been proposed. ${ }^{6} 8$

With spectral Doppler it is possible to evaluate the type of flow in the synovium-that is, low peripheral resistance versus high peripheral resistance. The degree of peripheral resistance is expressed numerically by the resistive index (RI). ${ }^{9}$ In this way it is possible to obtain objective information on the quality of flow in the synovium as a supplement to the estimation of vascularisation by colour pixels. Preliminary data have indicated low resistance flow and low RI values in pannus in RA. ${ }^{10}$ The dramatic effect of intra-articular injections of glucocorticosteroid has been known since $1951,{ }^{11}$ and as glucocorticosteroid treatment has previously been seen to diminish the power Doppler signal, ${ }^{45}$ it seems ideal to choose intra-articular treatment for an evaluation of the ability of a new measure to detect and quantify an antiinflammatory effect.

This study aimed at evaluating the quantitative response to glucocorticosteroid treatment by both colour and spectral Doppler in the diagnosis of inflammatory joint involvement in patients with RA and comparing the treatment with clinical estimates of the disease activity.

\section{PATIENTS AND METHODS \\ Patients}

Fifty one patients with rheumatoid arthritis were included (11 men, 40 women; mean age 63, range (29-93); mean disease duration 12 years, range $(1-42) ; 34$ had positive rheumatoid factor). All the patients needed an intra-articular glucocorticosteroid injection to treat a single joint flare

Abbreviations: $C D I$, colour Doppler image; $C R P, C$ reactive protein; DMARD, disease modifying antirheumatic drug; ESR, erythrocyte sedimentation rate; $\mathrm{HAQ}$, Health Assessment Questionnaire; MRI, magnetic resonance imaging; NSAID, non-steroidal anti-inflammatory drug; RA, rheumatoid arthritis; RI, resistive index; ROI, region of interest; US, ultrasound; VAS, visual analogue scale 

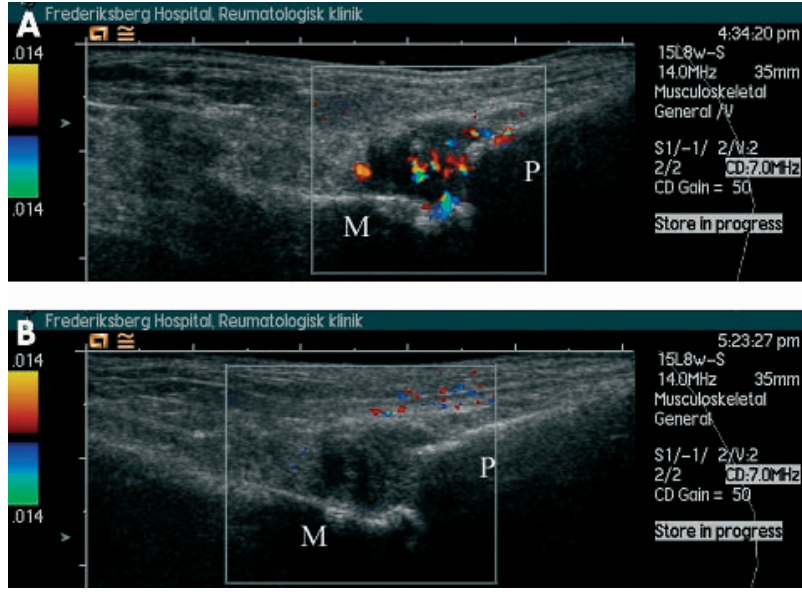

Figure 1 Longitudinal scan of the dorsal side of the right second metatarsophalangeal joint (A) before and (B) four weeks after intraarticular injection with $40 \mathrm{mg}$ methylprednisolone. The synovial tissue is seen as a hypoechoic (dark) mass swelling from the joint. The metacarpal bone is marked " $M$ " and the phalangeal bone " $\mathrm{P}$ ". The phalangeal bone is subluxated dorsally. In the bottom image the coloured pixels have completely disappeared - a sign of reduced flow in the treated region. The few scattered extrasynovial colour pixels in the bottom image are generated by noise.

up. The joints included 30 wrists, 9 elbows, 5 proximal interphalangeal joints, 2 metacarpophalangeal joints, 1 metatarsophalangeal joint, 3 talocrural joints, and 1 sternoclavicular joint. For at least three months before the study all patients were receiving stable disease modifying antirheumatic drug (DMARD) and non-steroidal anti-inflammatory drug (NSAID) treatment. During the observation period of four weeks their drugs were unchanged and no further injections were given. None of the patients received oral glucocorticosteroid treatment in doses larger than $7.5 \mathrm{mg}$ daily. The patients fulfilled the 1987 American College of Rheumatology criteria for RA. ${ }^{12}$

Clinical evaluation included pain in the target joint on a $100 \mathrm{~mm}$ visual analogue scale (VAS), Health Assessment Questionnaire (HAQ), morning stiffness in minutes, and the degree of swelling and tenderness of the target joint graded from 0 to 3. Blood samples were obtained for the determination of erythrocyte sedimentation rate (ESR) and $C$ reactive protein (CRP).

\section{Ultrasound}

An Acuson Sequoia ultrasound system (Mountainview, California, USA) was used for the ultrasound examinations using a $15 \mathrm{MHz}$ linear array transducer. The target joint was scanned at predefined transducer positions for that particular joint and the synovial membrane was examined longitudinally and transversally. Blood flow in the synovial membrane was visualised with colour Doppler ultrasonography. The colour Doppler settings were the same for all joints and all patients with a gain setting just below the noise level using our set up for low flow-Nyquist limit $+/-0.014 \mathrm{~m} / \mathrm{s}$ and $7 \mathrm{MHz}$ Doppler frequency. With this set up all the colour pixels in the image correspond to motion-that is, blood flow. Quantitative estimation of the vascularisation in the synovial membrane was performed using the selected colour Doppler image (CDI) with maximum colour activity. At present the colour Doppler is as sensitive as the power Doppler on the Sequoia. The digitally stored CDI in DICOM format was transferred to a processing program (Corel PhotoPaint 7) (Corel Corporation, Ottawa, Ontario, Canada). The synovium was traced, indicating the region of interest (ROI). On the US image the synovium appears as a predominantly hypoechoic mass covering the bony surfaces adjacent to the joint. By using a colour recognition function the number of colour pixels as well as the total number of pixels in the ROI were counted. ${ }^{8}$ The number of colour pixels was then expressed in relation to the total amount of pixels in the ROI as the colour fraction. The total number of pixels ( = synovial area) was also noted separately, as an estimate of the synovial membrane volume. The images were evaluated with the sequence and name of the patient "blinded".

With colour Doppler as guidance, the spectral Doppler sampling area was placed over an intrasynovial artery and the spectrum was obtained. Three random synovial arteries were sampled in this way. The ultrasound unit traced the Doppler spectra electronically and identified the cardiac cycles as well as peak systolic flow and end diastolic flow. The unit then calculated the RI as (peak systolic flow - end diastolic flow)/peak systolic flow. In this paper we used a mean RI value obtained from three different synovial artery values as described above. Low RI values correlate with a low peripheral resistance, indicating an increased tissue perfusion. ${ }^{13-15}$ When spectral Doppler measurements could not be measured owing to the lack of inflammatory activity in the examined joint, the RI was defined as 1.00, assuming the resistance in the synovial

\begin{tabular}{llll} 
Table 1 Changes in clinical and ultrasound parameters during the observation period \\
\hline & Baseline & $\mathbf{4}$ Weeks & $\begin{array}{l}\text { Mean change and } \\
\text { significance }\end{array}$ \\
\hline & $0.21(0.16)$ & $0.10(0.13)$ & $0.11(0.19)^{* * *}$ \\
\hline $\begin{array}{l}\text { Pixel fraction } \\
\text { Total pixels }\end{array}$ & $14721.5(12043.5)$ & $10169.9(7567.2)$ & $4551.56(9389.67)^{* *}$ \\
RI mean & $0.71(0.12)$ & $0.79(0.17)$ & $-0.078(0.2)^{* *}$ \\
VAS (target joint) & $53.9(28.0)$ & $35.2(26.2)$ & $18.7(25.7)^{* * *}$ \\
HAQ & $16.1(8.3)$ & $13.7(9.3)$ & $2.4(5.4)^{*}$ \\
ESR & $19.4(14.79)$ & $18.8(15.9)$ & $0.5(12.8)^{\text {Ns }}$ \\
CRP & $21.2(21.6)$ & $16.2(16.4)$ & $5.0(15.7)^{*}$ \\
$\begin{array}{l}\text { Morning stiffness (min), median } \\
\text { (range) }\end{array}$ & $30(0-300)$ & $5(0-300)$ & $25^{*}$ \\
$\begin{array}{l}\text { Patient's global assessment, } \\
\text { median (range) }\end{array}$ & $3(2-4)$ & $3(1-4)$ & $0^{*}$ \\
$\begin{array}{l}\text { Tenderness of the target joint, } \\
\text { median (range) }\end{array}$ & $2(0-3)$ & $1(0-3)$ & $1^{* *}$ \\
$\begin{array}{l}\text { Swelling of the target joint, } \\
\text { median (range) }\end{array}$ & $2(0-3)$ & $1(0-3)$ & $1^{* * *}$ \\
\hline
\end{tabular}

${ }^{*} p \leqslant 0.05 ;{ }^{* *} p \leqslant 0.01 ;{ }^{* * *} p \leqslant 0.001$.

Results are shown as mean (SD) unless indicated otherwise. 
arteries to be the same as in extrasynovial musculoskeletal tissue.

The synovium was noted as non-inflamed when no pixels were present in the ROI.

After four weeks the measurements were repeated on the same joint using the predefined positioning of the probe.

\section{Injections}

All injections were carried out guided by ultrasound. The ultrasound probe was held just outside the disinfected area but with a clear view of the area of interest. Using the "notouch" technique, the needle was then inserted into the joint cavity visualised on ultrasound to ensure correct placement of the needle. The injection site was determined from the ultrasound image as the area with the most pronounced Doppler activity. The following injection with $40 \mathrm{mg}$ methylprednisolone was monitored and the injected fluid was seen
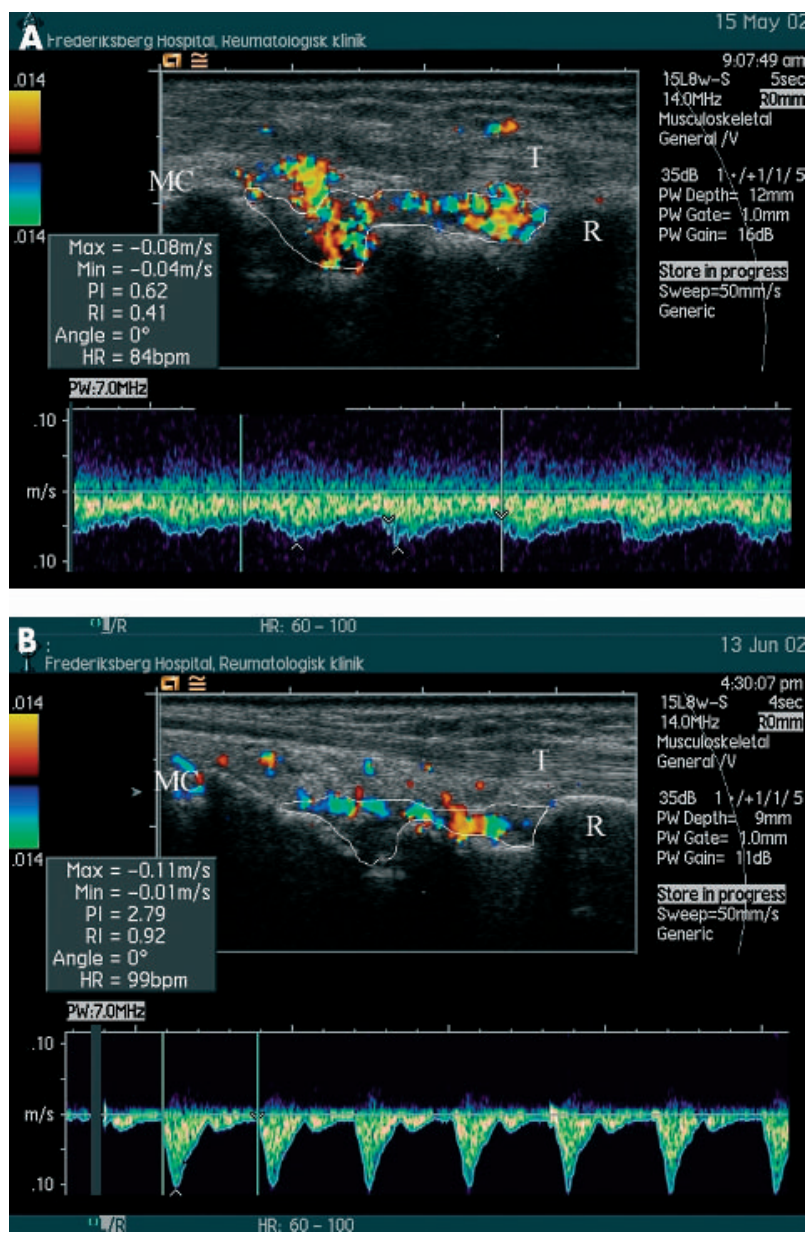

Figure 2 Longitudinal scan on the radial side of the left wrist showing the area with most activity (A) before and (B) four weeks after intraarticular injection with $40 \mathrm{mg}$ methylprednisolone. The radial bone $(R)$ is seen at the right side of the image and the metacarpus (MC) at the left. The extensor carpi radialis tendon is marked " $\mathrm{T}$ ". The synovium has been traced defining the ROI for the calculation of the colour fraction. The trace was made with the help of an extra image (not shown) where the colour had been removed, thereby allowing for definition of the border between synovium and extrasynovial tissue in the areas where it is covered with colour. In both images the Doppler gate (the space between the two horizontal lines on the dotted vertical Doppler line) has been placed over an intrasynovial artery and the resulting spectral curve is shown beneath. The ultrasound unit has traced the spectrum, defined maximum systolic value and end diastolic value, and displayed RI 0.41 and 0.92 ) in the data boxes. There is a reduction in colour fraction and an increase in peripheral resistance (increase in RI) as signs of reduced flow to the treated region. distributing in the joint cavity. In some of the finger joints it was impossible to inject all $40 \mathrm{mg}$ of methylprednisolone because of lack of space. In these situation as much as possible was injected ( $\geqslant 20 \mathrm{mg}$ ).

\section{Statistics}

Statistical analysis was performed using the SPSS 10.0 program.

Student's $t$ test for paired samples was used for continuous data, which include ESR, CRP, the pixel fraction, the total number of pixels, and mean RI. We also chose to regard HAQ and VAS as continuous data in this study. As model control the difference between the baseline and follow up visits was tested for normality ( 1 -sample Kolmogorow-Smirnoff test). The CRP had a borderline normal distribution, but the choice of test had no influence on the statistical result. Wilcoxon signed rank sum test was used for categorical data, including morning stiffness and assessment for tender and swollen joints. For the correlation between the US parameters and the clinical evaluation Pearson's test was used.

For statistical reasons we needed a value for RI even when no vessels were detectable in the synovium and we defined it as 1.00 in those situations. Level of significance was chosen at $\mathrm{p} \leqslant 0.05$

\section{RESULTS}

Table 1 shows the changes in clinical, biochemical, and ultrasound parameters during the observation period. There was a significant change in the global disease activity parameters such as CRP, morning stiffness and HAQ, after the intra-articular treatments, while the ESR remained stable. The local disease activity parameters such as synovial area, VAS, tender and swollen joint assessment of the target joint all showed significant changes after the treatment

For the ultrasound parameters $41 / 51$ had a significant decrease in colour fraction after the treatment, whereas only $32 / 51$ had a significant increase in mean RI. The synovial volume assessed by the total amount of grey pixels decreased in $38 / 51$.

The presence or absence of a positive rheumatoid factor had no impact on the treatment response. We found a significant correlation between the mean RI and the colour fraction $(r=-0.32, \mathrm{p}=0.02)$, between the pixel fraction and the tender joint assessment of the target joint $(r=0.6$, $\mathrm{p}=0.02)$ and the ESR $(r=0.34, \mathrm{p}=0.02)$, and between mean RI and the VAS $(r=-0.38, \mathrm{p}=0.02)$. No other significant correlations were found.

\section{DISCUSSION}

This study showed a significant effect of intra-articular glucocorticosteroid injections on the quantitative ultrasound measurements of synovial disease activity. The effect was demonstrated on both the synovial vascularisation (as measured by the colour fraction) (fig 1) and in the flow profile indicated by the increasing RI values due to a higher peripheral resistance (fig 2). Our findings of a decrease in colour fraction are in accordance with those of other studies. ${ }^{45}$ Also the synovial volume expressed by the total amount of pixels in ROI, showed a significant decrease by $31 \%$ after anti-inflammatory treatment, which is in agreement with other studies. ${ }^{16}{ }^{17}$ The ultrasound changes in our study paralleled the clinical evaluation by the clinician and the subjective pain evaluation, morning stiffness, global evaluation, and HAQ by the patient.

The dramatic anti-inflammatory effect of intra-articular treatment with glucocorticosteroids has been known since 1951, when Hollander et al first injected hydrocortisone acetate intrasynovially into patients with RA and found that they acted quickly on pain and joint swelling in $90 \%$ of the 
treated joints. ${ }^{11}$ Since then, intra-articular injections have become standard adjuvant treatment to normal NSAID and/ or DMARD treatment of RA. One or several injections into the same joint may improve the patient's condition significantly by decreasing joint pain and swelling, leading to improved function. The effect of intra-articular treatment varies from patient to patient, but may last for years. ${ }^{18}{ }^{19}$ Intra-articular treatment has the advantage over oral glucocorticosteroid treatment in that the systemic effects of the drug used are minimal, though they may be seen in some patients. ${ }^{20}$

Doppler US is a relatively new tool in the investigation of local inflammation. ${ }^{1021}$ The Doppler signals are a reflection of the number of red blood cells that pass the transducer and the speed with which they pass. The colour pixels are therefore an estimate of the relative amount of moving blood in the investigated area. ${ }^{22} 23$ The appearance of colour pixels in a synovial membrane is a sign of an increase in the relative amount of moving blood, because colour pixels are rarely seen in normal joints. The number of vessels does not correspond with the number of colour pixels visualised by Doppler ultrasound but rather with the area of the already existing vessels. ${ }^{24}$ The colour pixels may therefore be interpreted as a sign of hyperaemia. ${ }^{5}$ Although Doppler ultrasonography does not depict hyperaemia itself, ${ }^{25}$ it depicts the increase in flow that is a part of the inflammatory process. Spectral as well as colour Doppler seems to offer an objective and easily obtainable estimate of the degree of synovial inflammation, which may be used both in clinical trials and in daily clinical practice. The RI (resistive index, resistance index, or Pourcelot index ${ }^{9}$ ) is a numerical value of the amount of diastolic flow and is directly proportional to the peripheral vascular resistance. The RI has been used in obstetrics and in nephrology to give a numerical value for the diastolic component in the umbilical arteries and arteries of the transplanted kidney. ${ }^{26}$ In normal resting musculoskeletal tissues flow is seen with colour or power Doppler in muscles, connective tissue, seldom in joints, and never in tendons. When assessed by spectral Doppler, no diastolic flow is found in the resting tissues and, consequently, the RI is 1.00. In rheumatology low values of RI indicate disease, which has been observed in previous studies, ${ }^{78} 102829$ although a definite cut off level for abnormality remains to be established.

Colour and power Doppler have been used to identify the hyperaemia associated with inflammation..$^{21} 2430$ The use of the colour fraction as an anti-inflammatory measure has in other studies been evaluated after treatment with glucocorticosteroids ${ }^{4}$ and has also been found to be comparable with the changes in histology and MRI. ${ }^{230}$ The number of colour pixels in the synovium thus seems to correlate with the disease activity. The determination of the colour fraction within the ROI as described in this paper is suggested as an objective measure. The intra- and interobserver intraclass correlation coefficients for this method have been determined in an earlier study as $0.82-0.97$ and 0.82 , respectively. ${ }^{8}$ Alternatively, as described in other studies hyperaemia may be graded $0-3$ based on the subjective estimate of the examiner, although the reproducibility has not yet been determined. ${ }^{4}$ A comparison of scoring systems remains to be undertaken, but a standardised quantitative measurement will be of advantage in longitudinal studies. The RI, on the other hand, is determined by the ultrasound machine and is not dependent on the level of experience of the investigator. We found that the decrease in colour pixel fraction after treatment was paralleled by an increase in vascular resistance (RI). Both changes indicate a diminished flow in the synovium and thus a decrease in inflammatory activity. It may be speculated that the RI indicates changes in inflammation despite the presence of flow-that is, when pixel evaluation is more or less unchanged. Only weak or no associations have been observed between imaging parameters and estimates of the global clinical status. ${ }^{28}{ }^{81}$ This may be due to differences in the general state of the disease and the local disease activity in, for example, a finger or a wrist joint. In our study, the US parameters (colour fraction and the mean RI) correlated with ESR, VAS, and tender joint assessment.

\section{CONCLUSION}

This study shows that the colour fraction can detect changes in the synovial vascularisation due to treatment. It also suggests that RI may be an objective measure of inflammation and treatment response in patients with RA. The RI measure may be used as an alternative or adjunct to the estimation of the colour fraction and has the advantage of being automated.

\section{ACKNOWLEDGEMENTS}

The study was supported by the OAK foundation

\section{Authors' affiliations}

L Terslev, S Torp-Pedersen, E Qvistgaard, B Danneskiold-Samsoe, H Bliddal, The Parker Institute, Department of Rheumatology,

Frederiksberg Hospital, DK 2000 Frederiksberg, Denmark

\section{REFERENCES}

1 Backhaus M, Kamradt T, Sandrock D, Loreck D, Fritz J, Wolf KJ, et al. Arthritis of the finger joints: a comprehensive approach comparing conventional radiography, scintigraphy, ultrasound, and contrast-enhanced magnetic resonance imaging. Arthritis Rheum 1999:42:1232-45.

2 Szkudlarek M, Court-Payen, Strandberg C, Klarlund M, Klausen T, Ostergaard $M$. Power Doppler ultrasonography for assessment of synovitis in the metacarpophalangeal joints of patients with rheumatoid arthritis: a comparison with dynamic magnetic resonance imaging. Arthritis Rheum $2001 ; 44: 2018-23$.

3 Resnick D. Common disorders of synovium-lined joints: pathogenesis, imaging abnormalities, and complications. AJR Am J Roentgenol 1988;151:1079-93.

4 Stone M, Bergin D, Whelan B, Maher M, Murray J, McCarthy C. Power Doppler ultrasound assessment of rheumatoid hand synovitis. J Rheumatol 2001;28:1979-82.

5 Newman JS, Laing TJ, McCarthy CJ, Adler RS. Power Doppler sonography of synovitis: assessment of therapeutic response-preliminary observations. Radiology 1996; 198:582-4.

6 Hau M, Kneitz C, Tony HP, Keberle M, Jahns R, Jenett M. High resolution ultrasound detects a decrease in pannus vascularisation of small finger joints in patients with rheumatoid arthritis receiving treatment with soluble tumour necrosis factor alpha receptor (etanercept). Ann Rheum Dis 2002:61:55-8.

7 Terslev L, Torp-Pedersen S, Qvistgaard E, Kristoffersen H, Røgind H, Danneskiold-Samsøe B, et al. Effects of treatment with etanercept (Enbrel, TNRF:Fc ) on rheumatoid arthritis evaluated by Doppler ultrasonography. Ann Rheum Dis 2003:62:178-82.

8 Qvistgaard E, Rogind H, Torp-Pedersen S, Terslev L, Danneskiold-Samsoe B, Bliddal H. Quantitative ultrasonography in rheumatoid arthritis: evaluation of inflammation by Doppler technique. Ann Rheum Dis 2001;60:690-3.

9 Pourcelot L. L'examen Doppler des vaisseaux périphériques. Paris: AC-D Production, 1982.

10 Silvestri E, Martinoli C, Onetto F, Neumaier CE, Cimmino MA, Derchi LE. [Evaluation of rheumatoid arthritis of the knee with Doppler color]. Radiol Med (Torino) 1994;88:364-7.

11 Hollander JL. Hydrocortisone and cortisone injection into arthritic joints. Comparative effects of and use of hydrocortisone as a local antiarthritic agent. JAMA 1951;147:1629-35.

12 Arnett FC, Edworthy SM, Bloch DA, McShane DJ, Fries JF, Cooper NS, et al. The American Rheumatism Association 1987 revised criteria for the classification of rheumatoid arthritis. Arthritis Rheum 1988:31:315-24.

13 Hsu WH, Chiang CD, Chen CY, Kwan PC, Hsu JY, Hsu CP, et al. Color Doppler ultrasound pulsatile flow signals of thoracic lesions: comparison of lung cancers and benign lesions. Ultrasound Med Biol 1998;24:1087-95.

14 Bude RO, Rubin JM. Effect of downstream cross-sectional area of an arterial bed on the resistive index and the early systolic acceleration. Radiology 1999;212:732-8.

15 Adamson SL. Arterial pressure, vascular input impedance, and resistance as determinants of pulsatile blood flow in the umbilical artery. Eur J Obstet Gynecol Reprod Biol 1999:84:119-25.

16 Koski JM, Hermunen H. Intra-articular glucocorticoid treatment of the rheumatoid wrist. An ultrasonographic study. Scand J Rheumatol 2001;30:268-70.

17 Ostergaard M, Stoltenberg M, Gideon P, Sorensen K, Henriksen O, Lorenzen I. Changes in synovial membrane and joint effusion volumes after 
intraarticular methylprednisolone. Quantitative assessment of inflammatory and destructive changes in arthritis by MRI. J Rheumatol 1996;23:1151-61.

18 Neidel J, Boehnke M, Kuster RM. The efficacy and safety of intraarticular corticosteroid therapy for coxitis in juvenile rheumatoid arthritis. Arthritis Rheum 2002:46:1620-8.

19 McCarty DJ. Treatment of rheumatoid joint inflammation with triamcinolone hexacetonide. Arthritis Rheum 1972;15:157-73.

20 Emkey RD, Lindsay R, Lyssy J, Weisberg JS, Dempster DW, Shen V. The systemic effect of intraarticular administration of corticosteroid on markers of bone formation and bone resorption in patients with rheumatoid arthritis. Arthritis Rheum 1996;39:277-82

21 Newman JS, Adler RS, Bude RO, Rubin JM. Detection of soft-tissue hyperemia: value of power Doppler sonography. AJR Am J Roentgenol 1994;163:385-9.

22 Rubin JM, Adler RS, Fowlkes JB, Spratt S, Pallister JE, Chen JF, et al. Fractional moving blood volume: estimation with power Doppler US. Radiology 1995; 197:183-90

23 Rubin JM, Bude RO, Carson PL, Bree RL, Adler RS. Power Doppler US: a potentially useful alternative to mean frequency-based color Doppler US. Radiology 1994:190:853-6.

24 Schmidt WA, Volker L, Zacher J, Schlafke M, Ruhnke M, Gromnica-Ihle E. Colour Doppler ultrasonography to detect pannus in knee joint synovitis. Clin Exp Rheumatol 2000;18:439-44.
25 Cardinal E, Lafortune M, Burns P. Power Doppler US in synovitis: reality or artifact? Radiology 1996;200:868-9.

26 Skjoldbye B, Nielsen AH, Court-Payen, Norgaard N, Rasmussen F, Lokkegaard $\mathrm{H}$, et al. Perioperative Doppler ultrasonography: renal detection of renal graft perfusion. Scand J Urol Nephrol 1998;32:345-9.

27 Struijk PC, Ursem NT, Mathews J, Clark EB, Keller BB, Wladimiroff JW. Power spectrum analysis of heart rate and blood flow velocity variability measured in the umbilical and uterine arteries in early pregnancy: a comparative study. Ultrasound Obstet Gynecol 2001;17:316-21.

28 Troltzsch $M$. [Color Doppler study in patients with rheumatoid arthritis and scleroderma]. Z Rheumatol 1994;53:2-6.

29 Midiri M, lovane A, Finazzo M, Brancatelli G, Gallo C, Lagalla R. [Color Doppler-echo in rheumatoid arthritis with extra-articular location. Preliminary experience]. Radiol Med (Torino) 1999;98:123-6.

30 Walther M, Harms H, Krenn V, Radke S, Faehndrich TP, Gohlke F. Correlation of power Doppler sonography with vascularity of the synovial tissue of the knee joint in patients with osteoarthritis and rheumatoid arthritis. Arthritis Rheum 2001:44:331-8.

31 Klarlund M, Ostergaard M, Gideon P, Sorensen K, Jensen KE, Lorenzen I. Wrist and finger joint MR imaging in rheumatoid arthritis. Acta Radiol 1999:40:400-9

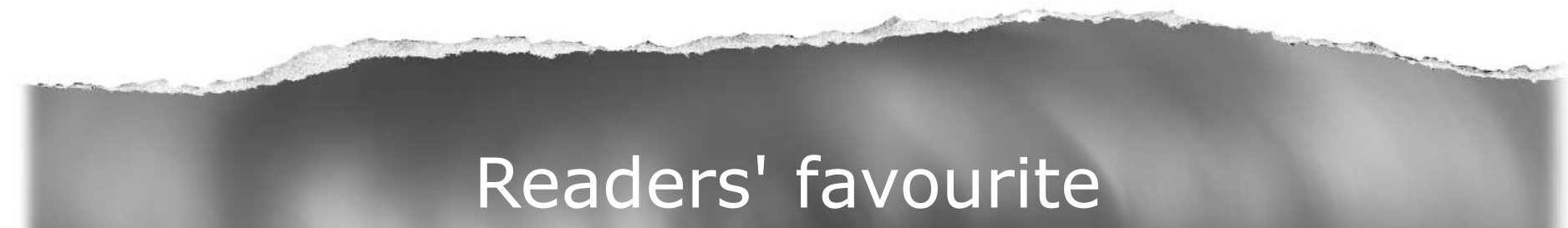

\section{Top 10}

Click on the "Top 10" button on the homepage to see which are the best read articles each month

www.annrheumdis.com 